\title{
VARIATIONAL PROBLEMS WITHIN THE CLASS OF SOLUTIONS OF A PARTIAL DIFFERENTIAL EQUATION
}

\author{
BY \\ ROBERT DELVER $\left({ }^{1}\right)$
}

\begin{abstract}
The subject of this paper is the optimization of a multiple integral over a domain $G$ of a function, containing as arguments the independent variables, the unknown function and its partial derivatives up to order $l$, within the class of all sufficiently smooth solutions in $G$ of a given partial differential equation of order greater than or equal to $2 l$. Necessary conditions in the form of a boundary value problem are derived. A physical application occurs in the control with boundary and initial conditions of a process in $G$ that is described by a specific partial differential equation.
\end{abstract}

I. Introduction. A classical problem in the calculus of variations is the optimization of a multiple integral over a domain $G$ of a function containing as arguments the independent variables, the unknown function and its partial derivatives up to order $l$. Usually the unknown function is required to be an element of the class of all functions that are 2 -times continuously differentiable defined in an open domain containing $G$.

The optimization problem that is dealt with in this paper differs from the one above in that the class of admissible functions to be considered is the collection of all sufficiently smooth solutions in $G$ of a given partial differential equation of order greater than or equal to $2 l$.

The most important points in this paper are the definition of the variational adjoint, the derivation of the variational adjoint boundary conditions, and the derivation of necessary conditions. The necessary conditions take the form of a boundary value problem.

Somewhat related to the problem of this paper are boundary control problems where the class of admissible functions is generated by varying controls in the right-hand side of a given partial differential equation and in certain boundary and initial conditions. A. Friedman [4] considers such a problem in boundary control

Received by the editors May 25, 1971.

AMS (MOS) subject classifications (1970). Primary 49B15, 49B25.

Key words and phrases. Calculus of variations, optimization problems, boundary control of partial differential equations, variational adjoint, associated boundary value problem, necessary conditions.

( ${ }^{1}$ ) This paper contains results from the author's doctoral thesis at the University of California in Berkeley, written under the direction of Professor M. H. Protter. 
theory for parabolic equations. In his paper a maximum principle is derived. It also contains a proof of the existence of an optimal solution for this problem. $\mathrm{K}$. A. Lurie, [5, Chapter 5], considers such a problem for elliptic equations in 2-dimensional Euclidian space. Other related control problems are studied in J. L. Lions [6]. This book gives many further references to the literature.

Chapter II contains a complete derivation of the necessary conditions for the optimization problem associated with the Laplace equation. This chapter gives an indication of the essentials in the later derivations. Also it gives a first motivation for the definition of the variational adjoint function and of the variational adjoint boundary conditions. The chapter contains a simple application.

In Chapter III an explicit form of Green's identity for higher order linear partial differential expressions is derived. This identity plays an important role in Chapter V. Due to the symmetric notation of the differential expression, the derivation given is analogous to that for ordinary differential equations.

Chapter IV contains lemmas to be used in the next chapter. The Lemmas IV, 1 and IV, $3 a$ and $b$ are purely technical. Lemma IV, 2 is somewhat more important and may find applications in other related problems.

Chapter $\mathrm{V}$ is the most important part of the paper. It contains a precise definition of the optimization problem, a definition of the variational adjoint function and of the variational adjoint boundary conditions. In Theorem V, 1 a special form of the variational adjoint boundary conditions is derived. In the next chapters, this result serves as a foundation for the formulation of the necessary conditions.

For the optimization problem associated with elliptic and parabolic equations necessary conditions in the form of a boundary value problem are derived in the last two chapters respectively. Each of these chapters depends completely on well-known existence theorems and on the results of Chapter V. Not only do these chapters each have a meaning of the ir own but together they constitute a method for the use of the variational adjoint in variational problems associated with other types of partial differential equations for which there is an existence the orem comparable with the ones in the last two chapters.

The following notation is used. $R^{\nu}$ is the $\nu$-dimensional Euclidian space. $G$ is an open bounded domain in $R^{\nu}$ with boundary $\partial G, B(a, b)$ is the open ball in $R^{\nu}$ with centre $a$ and radius $b . \sigma(0, b)$ is the intersection of $B(0, b)$ with the hyperplane $x_{\nu}=0$. In a sequence of elements, , denotes elements that are not written down. In a multiple product $\cdots$ denotes factors that are not written down. If $a$ and $b$ are integers then $i \in[a, b]$ denotes that $i$ takes one integer value in the interval $[a, b]$ and $a \leq i \leq b$ denotes that $i$ takes successively all integer values in $[a, b]$. If $A$ is an open set in $R^{\eta}, \eta$ is a positive integer, then $C^{k}(A)$ denotes the set of all $k$ times continuously differentiable functions defined on $R^{\eta}$ and 
$C^{k}(\bar{A})$ denotes the set of all elements of $C^{k}(A)$ that together with their derivatives of order less than or equal to $k$ can be extended to be continuous on $\bar{A}$. $\partial G \in C^{k}$ denotes that $\partial G$ is $k$ times continuously differentiable. If $\partial G \in C^{1}$ then $\mathbf{n}$ is the outward unit normal vector to $\partial G$. If $A \subset R^{\nu}$, then $\operatorname{nbh} A$ is an open set in $R^{\nu}$ that contains $A$. If $u \in C^{k}(G)$, then $\|u(x)\|_{k}$ denotes the sum of the supremums in $G$ of the absolute values of the function $u$ and all its derivatives of order less than or equal to $k_{\text {. If }} \alpha \in[1, \nu]$ then $D_{\alpha} u(x)$ denotes $\partial u(x) / \partial x_{\alpha}, u_{\alpha}$ is the same as $D_{a} u(x)$. If $\partial G \in C^{k}$ and if $u \in C^{k}(\mathrm{nbh} \partial G)$ then $(\partial / \partial \mathbf{n})^{k} u(x)$ is the $k$ th derivative of $u$ along $\mathbf{n}$ on $\partial G$.

Convention. Summation is taken over indices $\alpha_{i}$ if they are repeated in the same term. The summation indices $\alpha_{i}$ always run independently from 1 to $\nu$. If the first index in a sequence of indices has a serial number that is one bigger than that of the last index in the subscript (or superscript), then the quantity is interpreted as one without any subscript (or superscript). (E.g. if $j=2$ then $u_{, a_{3},-, a_{j}}$ must be read as $u_{\text {.) }}$ The indices $\alpha_{i}^{\prime}$ run independently from 1 to $\nu-1$. Otherwise the same convention is in force.

Results of this paper can be extended to the case of systems of partial differential equations. This investigation will be the subject of a later report.

The results of this paper for elliptic differential equations have been extended to functionals with derivatives up to the order of the differential equation and nonuniquely solvable Dirichlet problem. For elliptic differential equations with uniquely solvable Dirichlet problems the same techniques have been applied to get necessary conditions for optimization problems with control variables in the right hand side of the differential equation and in the boundary conditions. See [10].

II. Necessary conditions for the optimization problem associated with the Laplace equation. Let $G$ be a bounded domain in $R^{\nu}$ with boundary $\partial G$ of class $C^{2+a}$. In this chapter $\alpha$ is the exponent of Hölder continuity, $\alpha \in(0,1)$. For $f \in C^{a}(G)$ the class of admissible functions $W$ is defined by

$$
\begin{array}{cl}
u \in W \text { iff } & \left(1^{\circ}\right) \quad \Delta u(x)=f(x), \quad x \in G, \\
& \left(2^{\circ}\right) \quad u \in C^{2+a}(\bar{G}) .
\end{array}
$$

A functional $J$, defined on $W$, is given by

$$
J(u)=\int_{G} F(x, u(x), D u(x)) d V, \text { with } F \in C^{2+\alpha}\left(R^{1+2 \nu}\right) .
$$

Lemma II.l. If $J$ has a relative extremum within $W$ for some $u \in W$ then the principal linear part of the variation of $J, \delta J(u ; \delta u)$, given in (1.3), vanishes for all $\delta u \in W_{H}$, given in (1.4).

$$
\delta J(u ; \delta u)=\int_{G}\left\{\delta u \frac{\partial F}{\partial u}+\sum_{i=1}^{\nu} \delta u_{, i}\left(\frac{\partial F}{\partial u}, i\right)\right\} d V .
$$




$$
\begin{aligned}
\delta u \in W_{H} \text { iff } & \left(1^{\circ}\right) \quad \Delta \delta u(x)=0, \quad x \in G, \\
& \left(2^{\circ}\right) \quad \delta u(x) \in C^{2+a}(\bar{G}) .
\end{aligned}
$$

Somewhat more in general the definition of a relative extremum and the proof of the lemma are given in Chapter $\mathrm{V}$.

Existence Theorem (see e.g. [7]). Assume that $f \in C^{a}(\bar{G}), g \in C^{2+a}(\partial G)$, and that $\partial G \in C^{2+a}$, then the Dirichlet problem $\Delta u(x)=f(x), x \in G$; and $u(x)=g(x), x \in \partial G$, admits a unique solution which is of class $C^{2+a}(\bar{G})$.

Now the necessary condition $\delta J(u ; \delta u)$ vanishes for all functions $\delta u$ that satisfy (1.4) will be put in the form of a boundary value problem. After integration by parts of (1.3) one obtains

$$
\delta J(u ; \delta u)=\int_{G} \delta u(x)[F]_{, u} d V+\int_{\partial G}\left\{\delta u(x) \sum_{i=1}^{\nu}\left(n_{i} \frac{\partial F}{\partial u}, i\right)\right\} d S
$$

here

$$
[F]_{, u}=\frac{\partial F}{\partial u}-\sum_{i=1}^{\nu} D_{i}\left(\frac{\partial F}{\partial u, i}\right) \quad \text { (Euler expression). }
$$

In the classical problem of the calculus of variations, where the class of admissible functions is $C^{2}(\bar{G})$, one obtains at this point from some variational arguments that the condition $\delta J(u ; \delta u)=0$, for all $\delta u \in C^{2}(\bar{G})$, is equivalent with

$$
\begin{aligned}
& {[F]_{, u}=0, \quad \text { for } x \in G \text { (Euler equation), }} \\
& \sum_{i=1}^{\nu} n_{i} \frac{\partial F}{\partial u_{, i}}=0, \quad \text { for } x \in \partial G \text { (transversality condition). }
\end{aligned}
$$

In the problem that is considered here $\delta u$ satisfies (1.4). In consequence $u$ can only be varied in $G$ if it is varied on $\partial G$. This indicates that it may be useful to transform $\delta J(u ; \delta u)$ into a boundary integral. To this end let $v$ be any function that satisfies

$$
\begin{aligned}
& \Delta v(x)=[F]_{, u}, \quad x \in G, \\
& v \in C^{2}(\bar{G}) .
\end{aligned}
$$

From Green's identity, from (1.4) and from (1.7) one obtains

$$
\begin{aligned}
& \int_{G} \delta u(x)[F], u=\int_{G}\{\delta u(x) \Delta v(x)-v(x) \Delta(\delta u(x))\} d V \\
& =\int_{\partial G}\left\{\delta u(x)\left(\frac{\partial}{\partial \mathbf{n}}\right) v(x)-v(x)\left(\frac{\partial}{\partial \mathbf{n}}\right) \delta u(x)\right\} d S .
\end{aligned}
$$

Substitution of this result in (1.5) yields

$$
\delta J(u ; \delta u)=\int_{\partial G}\left\{\delta u(x)\left\{\left(\frac{\partial}{\partial \mathbf{n}}\right) v(x)+\sum_{i=1}^{\nu} n_{i} \frac{\partial F}{\partial u_{, i}}\right\}-\left\{\left(\frac{\partial}{\partial \mathbf{n}}\right) \delta u(x)\right\} v(x)\right\} d S .
$$


If $\delta u$ is restricted to $W_{H}$, it follows from the existence theorem for the Dirichlet problem that $\delta u(x)$, for $x \in \partial G$, still can be chosen arbitrarily in $C^{2+a}(\partial G)$, while the following boundary condition can be chosen for $v$.

$$
v(x)=0, \quad \text { for } x \in \partial G .
$$

After substitution of (1.9) into (1.8) and using the freedom of $\delta u$ one obtains that $\delta J(u ; \delta u)=0$ for all $\delta u \in W_{H}$, if the solution of (1.7), (1.9) satisfies also the boundary condition:

$$
\left(\frac{\partial}{\partial \mathbf{n}}\right) v(x)=-\sum_{i=0}^{\nu} n_{i} \frac{\partial F}{\partial u_{, i}}, \quad x \in \partial G .
$$

The results can be summarized as follows:

Theorem II.1. If $J$ bas a relative extremum within $W$ for some $u \in W$ then the boundary value problem (1.11), evaluated for this $u$, is satisfied.

$$
\begin{aligned}
& \Delta u(x)=f(x), \quad x \in G, \\
& \Delta v(x)=[F]_{, u}, \quad x \in G, \\
& v(x)=0, \quad x \in \partial G, \\
& \left(\frac{\partial}{\partial \mathbf{n}}\right) v(x)=-\sum_{i=1}^{\nu} n_{i} \frac{\partial F}{\partial u_{, i}}, \quad x \in \partial G .
\end{aligned}
$$

Instead of the existence theorem for the Dirichlet problem an existence theorem for the Neumann problem could be used to derive the same theorem. In that case the condition (1.10) is chosen and substituted into (1.8), while, from the freedom of $(\partial / \partial n) \delta u$, one obtains now (1.9) as a necessary condition.

Sufficient motivation has been given so that some terminology may be introduced. A solution of (1.7) that satisfies the boundary conditions

(1.12) $\delta J(u ; \delta u)$, as given in (1.8), vanishes for all functions $\delta u \in C^{2}$ (nbh $\partial G$ ) is called a variational adjoint function of $u$ with respect to $\Delta$ and $J$. The boundary conditions (1.12) are called variational adjoint boundary conditions. It is clear that they are equivalent with (1.9) and (1.10) together. (More generally, this is the content of Theorem V.1.) Depending on the existence theorem that is used, chosen and imposed variational adjoint boundary conditions are distinguished. With respect to the Dirichlet problem (1.9) is chosen and (1.10) is imposed. With respect to the Neumann problem these are reversed.

Now Theorem II. 1 can be phrased as: If $J$ has a relative extremum within $W$ for some $u \in W$, then $u$ has a variational adjoint function, with respect to $\Delta$ and $J$.

The boundary value problem (1.11) is called the associated boundary value problem.

The chapter will be concluded with an application of Theorem II.1. 
Example. Let $G, W$ and $f$ be defined as before in this chapter.

Assertion. The functional $J(u)=\int_{G} 1 / 2 \Sigma_{i=1}^{\nu}\left(u_{, i}\right)^{2} d V$ bas a relative minimum within $W$ for any $u$ that satisfies $\Delta u(x)=f(x), x \in G$; and $u(x)$ is constant on $\partial G$.

Proof. The associated boundary value problem takes the form

$$
\begin{aligned}
& \Delta u(x)=f(x), \quad x \in G, \\
& \Delta v(x)=-\Delta u(x), \quad x \in G, \\
& v(x)=0, \quad x \in \partial G, \\
& \left(\frac{\partial}{\partial \mathbf{n}}\right) v(x)=-\left(\frac{\partial}{\partial \mathbf{n}}\right) u(x), \quad x \in \partial G .
\end{aligned}
$$

So that

$$
\begin{aligned}
& \Delta(u(x)+v(x))=0, \quad x \in G, \\
& \left(\frac{\partial}{\partial \mathbf{n}}\right)(u(x)+v(x))=0, \quad x \in \partial G .
\end{aligned}
$$

The solution of (1.14) is $u(x)+v(x)=C$, for $x \in \bar{G}$, where $C$ is any constant. As $v$ vanishes on $\partial G$ one obtains the necessary conditions $u(x)=C$, for $x \in \partial G$. That this condition is sufficient for a relative minimum within $W$ follows from $J(u+\delta u)-J(u)>0$, if $u \in W ; u(x)=C$, for $x \in \partial G, u+\delta u \in W$. With this condition, writing $\cdot$ for inner product and $\nabla$ for the gradient, this is proved by

$$
\begin{aligned}
2\{J(u & +\delta u)-J(u)\} \\
& =\int_{G}\{\nabla(u(x)+\delta u(x)) \cdot \nabla(u(x)+\delta u(x))-\nabla u(x) \cdot \nabla u(x)\} d V \\
& =\int_{G}\{\nabla \delta u(x) \cdot \nabla \delta u(x)+2 \nabla u(x) \cdot \nabla \delta u(x)\} d V \\
& =\int_{G}\{\nabla \delta u(x) \cdot \nabla \delta u(x)+2 \nabla \cdot(u(x) \nabla \delta u(x))\} d V \\
& =\int_{G}\{\nabla \delta u(x) \cdot \nabla \delta u(x)\} d V+2 C \int_{\partial G}\left(\frac{\partial}{\partial \mathbf{n}}\right) \delta u(x) d S \\
& =\int_{G}\{\nabla \delta u(x) \cdot \nabla \delta u(x)\} d V>0 .
\end{aligned}
$$

III. Green's identity. In this chapter an explicit form of Green's identity will be derived for a linear partial differential expression of order $m$ in $\nu$ independent variables with real coefficients.

A differential expression $L$ and its formal adjoint $L^{*}$ are defined by

$$
\begin{aligned}
L & =\sum_{k=0}^{m} A(x)^{a_{1},-, a_{k}} D_{a_{1}} \cdots D_{a_{k}}, \quad x \in G . \\
L^{*} & =\sum_{k=0}^{m}(-1)^{k} D_{a_{1}} \cdots D_{a_{k}} A(x)^{a_{1},-, a_{k}}, \quad x \in G .
\end{aligned}
$$


The coefficients $A(x)^{\alpha}{ }^{1,-, a_{k}}$ are assumed to be of class $C^{k}(\Omega), \Omega$ is an open domain in $R^{\nu}$ containing $\bar{G}$, and symmetric in the indices $\alpha_{1},-, \alpha_{k}$, for $1 \leq k \leq m$. For two functions $u \in C^{m}(\Omega), v \in C^{m}(\Omega)$ and a domain $G$ with $\partial G \in C^{1}$, the Green's identity is given by

$$
\int_{G}\left\{u(x) L v(x)-v(x) L^{*} u(x)\right\} d V
$$

$$
=\int_{\partial G}\left\{\sum_{i=0}^{m-1} \sum_{j=0}^{m-i-1} u_{, \alpha_{1}, \ldots, \alpha_{i}, a_{i+1},-, a_{i+j}} P(i ; j){ }^{a_{1},-, a_{i+j}}\right\} d S .
$$

For $1 \leq i \leq m-1 ; 1 \leq j \leq m-i-1 ; x \in \partial G$, the functions $P(i ; j)^{\alpha_{1},-, \alpha_{i+j}}$ are defined by

$$
P(i ; j)^{\alpha_{1},-, a_{i+j}}=\sum_{k=i+j+1}^{m}(-1)^{k-j-1}\left(\begin{array}{c}
k-j-1 \\
i
\end{array}\right) A_{, a_{i+j+1},-, a_{k-1}}^{a_{1},-a_{k}} n_{a_{k}}
$$

Proof. From the product formula for differentiation one obtains, for $k \in[1, m]$

$$
\begin{aligned}
& D_{a_{k}}\left\{\sum_{b=0}^{k-1}(-1)^{b}\left\{u A^{a_{1}, \ldots, a_{k}}\right\}, a_{1, \ldots, a_{b}}{ }^{,}, a_{b+1}, \ldots, a_{k-1}\right\} \\
& =\sum_{b=0}^{k-1}(-1)^{b}\left\{u A^{a_{1},-, a_{k}}\right\}, a_{1}, \ldots, a_{b}^{v}, a_{b+1}, \ldots, a_{k} \\
& +\sum_{b=0}^{k-1}(-1)^{b}\left\{u A^{a_{1},-, a_{k}}\right\}, a_{1}, \ldots, a_{b}, a_{k}^{v}, a_{b+1}, \ldots, a_{k-1} \\
& =u A^{a_{1}, \ldots, a_{k}} v_{, a_{1}, \ldots, a_{k}}-(-1)^{k} v\left\{u A^{a_{1}, \ldots, a_{k}}\right\}, a_{1}, \ldots, a_{k} \text {. }
\end{aligned}
$$

For $k \geq 2$, the last identity is true because, by virtue of the symmetry of the functions $\bar{A}^{a_{1},-, a_{k}}$ in the indices, (1.6) and (1.7) cancel each other.

$$
\begin{aligned}
& \sum_{b=1}^{k-1}(-1)^{b}\left\{u A^{a_{1}, \ldots, a_{k}}\right\}, a_{1}, \ldots, a_{b}{ }^{v}, a_{b+1}, \ldots, a_{k}, \\
& \sum_{b=0}^{k-2}(-1)^{b}\left\{u A^{a_{1}, \ldots, a_{k}}\right\}, a_{1}, \ldots, a_{b}, a_{k}^{v}, a_{b+1}, \ldots, a_{k-1} .
\end{aligned}
$$

Because of the same symmetry (1.8), Leibnitz rule, can be derived analogously to that for functions of one independent variable $(k \in[1, m], b \in[0, k])$.

$$
\left\{u A^{a_{1}, \ldots, a_{k}}\right\}, a_{1, \ldots}, a_{b}=\sum_{i=0}^{b} u^{,}, a_{1,-, a_{i}}\left(\begin{array}{l}
b \\
i
\end{array}\right) A^{a_{1,-, a_{k}}, a_{i+1, \ldots, a_{b}}} \text {. }
$$

From (1.1), (1.2), (1.5) and (1.8) one obtains, for $x \in \Omega$, 


$$
\begin{aligned}
& u(x) L v(x)-v(x) L^{*} u(x) \\
& \quad=\sum_{k=1}^{m} D_{a_{k}}\left\{\sum_{b=0}^{k-1}(-1)^{b}\left\{\sum_{i=0}^{b} u_{, a_{1}, \ldots, a_{i} \backslash}\left(\begin{array}{l}
b \\
i
\end{array}\right) A_{, a_{i+1}, \ldots, a_{b}}^{a_{1}, a_{k}} v_{, a_{b+1}, \ldots, a_{k-1}}\right\} .\right.
\end{aligned}
$$

From an integration by parts, using this result, one obtains

$$
\begin{aligned}
& \int_{G}\left\{u(x) L v(x)-v(x) L^{*} u(x)\right\} d V \\
&=\int_{\partial G}\left\{\sum_{k=1}^{m} \sum_{b=0}^{k-1} \sum_{i=0}^{b}(-1)^{b} u_{, a_{1},-, a_{i}}\right. \\
&\left.\qquad\left(\begin{array}{l}
b \\
i
\end{array}\right) A_{, a_{i+1}, \ldots, a_{b}, a_{b+1}, \ldots, a_{k-1} n_{a_{k}}}{ }^{a_{1}, a_{k}}\right\} d S .
\end{aligned}
$$

Now, in order to get the more convenient structure of (1.3), (1.4), the summation must be rearranged.

Letting $j=k-b-1$, the integrand of the boundary integral in (1.10) becomes

$$
\sum_{k=1}^{m} \sum_{j=0}^{k-1} \sum_{i=0}^{k-j-1} u_{, a_{1}, \ldots, a_{i}}(-1)^{k-j-1}\left(\begin{array}{c}
k-j-1 \\
i
\end{array}\right) A_{, a_{i+1}, \ldots, a_{k-j-1}, a_{k-j},-, a_{k-1}}^{a_{a_{k}}}{ }^{n_{k}}
$$

For $k \in[1, m] ; j \in[0, k-1] ; i \in[0, k-j-1]$, it follows again from the symmetry that

$$
A_{, a_{i+1},-, a_{k-j-1}}^{a_{1}, a_{k-j},-, a_{k-1}}=A_{, a_{i+j+1},-, a_{k-1}, a_{i+1},-, a_{i+j}}^{a_{1},-a_{k}} .
$$

Using the last two results, the integrand of the boundary integral in (1.10) can be written as

$$
\sum_{k=1}^{m} \sum_{j=0}^{k-1} \sum_{i=0}^{k-j-1} u_{, a_{1}, \ldots, a_{i}, a_{i+1}, \ldots, a_{i+j}}(-1)^{k-j-1}\left(\begin{array}{c}
k-j-1 \\
i
\end{array}\right) A_{, a_{i+j+1}, \ldots, a_{k-1}}^{a_{1}, a_{k}} n_{a_{k}} .
$$

The set of inequalities $\{1 \leq k \leq m ; 0 \leq j \leq k-1 ; 0 \leq i \leq k-j-1\}$ is equivalent with $\{0 \leq i \leq m-1 ; 0 \leq j \leq m-i-1 ; i+j+1 \leq k \leq m\}$. Therefore the previous sum can be written as

$$
\sum_{i=0}^{m-1} \sum_{j=0}^{m-i-1} \sum_{k=i+j+1}^{m} u_{, a_{1},-, a_{i}{ }^{v} a_{i+1},-, a_{i+j}}(-1)^{k-j-1}\left(\begin{array}{c}
k-j-1 \\
i
\end{array}\right) A_{, a_{i+j+1}, \ldots, a_{k-1}}^{a_{1},-a_{k}} n_{a_{k}} .
$$

Substitution of this expression into (1.10) completes the proof.

\section{Technical lemmas.}

(1) Lemma IV.1. Let $G$ be a bounded domain in $R^{\nu}$ with $\partial G \in C^{k+b}, k \geq 0$, $b \geq 0$, and let $\Omega$ be an open domain in $R^{\nu}$ that contains $\bar{G}$. Then to every set of functions $g_{j}(x) \in C^{k+b-j}(\partial G), 0 \leq j \leq k$, there exists at least one function 
$w \in C^{k+b}(\Omega)$ that satisfies $(\partial / \partial \mathbf{n})^{j} w(x)=g_{j}(x)$, for $x \in \partial G$ and $0 \leq j \leq k$.

This lemma is a direct consequence of C. Miranda $[7,16, \mathrm{VI}]$ and $\mathrm{H}$. Whitney [8, Theorem 1 with Lemma 2].

(2) Lemma IV.2. Assume that

$\left(1^{\circ}\right) G$ is a bounded domain in $R^{\nu}$ with $\partial G \in C^{k}$,

$\left(2^{\circ}\right) w \in C^{k}(\bar{G})$,

$\left(3^{\circ}\right)(\partial / \partial \mathrm{n})^{j} w(x)=0$, for $x \in \partial G$ and $0 \leq j \leq k-1$.

Then

(i) $D^{\sigma} w(x)=0$, for $x \in \partial G$ and $0 \leq \sigma \leq k-1$,

(ii) $w_{, a_{1},-, a_{k}}=n_{a_{1}} \cdots n_{a_{k}}(\partial / \partial \mathrm{n})^{k} w(x)$, for $x \in \partial G$.

Proof. Let $\Omega$ be an open neighborhood of $\partial G$. Using $H$. Whitney [8, Lemma 2], $x$ is extended into $C^{k}(\Omega)$. Now normal derivatives of order less than or equal to $k$ can be defined by

$$
\begin{aligned}
\left(\frac{\partial}{\partial \mathbf{n}}\right)^{0} w(x) & =w(x), \quad x \in \Omega, \\
\left(\frac{\partial}{\partial \mathbf{n}}\right)^{j+1} w(x) & =\sum_{i=0}^{\nu} n_{i} D_{i}\left(\left(\frac{\partial}{\partial \mathbf{n}}\right)^{j} w(x)\right), \quad x \in \Omega .
\end{aligned}
$$

So that $(\partial / \partial n)^{j} w(x)$ is the restriction to $\partial G$ of a linear differential expression on $\Omega$ with principal part

$$
n_{a_{i}} \cdots n_{a_{j}} D_{a_{i}} \cdots D_{a_{j}} \text {. }
$$

Assume now that $D^{\sigma} w(x)=0$, for $0 \leq \sigma \leq j-1 \leq k-1$ and $x \in \partial G$. As the gradient on $\partial G$ of a function which is constant on $\partial G$ is normal to $\partial G$

$$
w_{, a_{1}, \ldots, a_{j}}=n_{a_{1}} a_{a_{2}, \ldots, a_{j}}, \quad x \in \partial G \text {. }
$$

Here the functions $a_{a_{2},-, a_{j}}$ are scalar functions defined on $\partial G$. As $w \in C^{k}(\Omega)$, the expressions in (2.3) are symmetric in the indices $a_{1},-, a_{j}$, so that for some $b \in[1, \nu]$,

$$
\begin{aligned}
& n_{b} a(x)_{a_{2}, \ldots, a_{j}}=n_{a_{2}} a(x)_{b a_{3}, \ldots, a_{j}} \\
& n_{b} a(x)_{b a_{3}, \ldots, a_{j}}=n_{a_{3}} a(x)_{b b a_{4}, \ldots, a_{j}}
\end{aligned}
$$

$$
n_{b} a(x)_{b,-, b, a_{j}}=n_{a_{j}} a(x)_{b,-, b} \text {. }
$$


Let $\partial G_{b}$ be the part of $\partial G$ where $n_{b}$ does not vanish then, for $x \in \partial G_{b}$, one obtains from (2.4)

$$
a_{a_{2,-, a_{j}}}=\left\{a_{b,-, b} / n_{b}^{b-1}\right\}_{a_{2}} \cdots n_{a_{j},} \quad x \in \partial G_{b} .
$$

From (2.5) and (2.2) one obtains

$$
w_{, a_{1},-, a_{j}}=n_{a_{1}} \cdots n_{a_{j}} \phi, \quad x \in \partial G .
$$

Here $\phi$ is a scalar function defined on $\partial G$. Still under the assumption $D^{\sigma} w(x)=0$, $0 \leq \sigma \leq j-1 \leq k-1, x \in \partial G$, one obtains from (2.2) and (2.6) that $\phi=(\partial / \partial n)^{j} w$, so that

(2.7) If $D^{\sigma} w(x)=0,0 \leq \sigma \leq j-1 \leq k-1$, then $w_{, a_{1},-, a_{j}}=n_{a_{1}} \cdots$ $n_{a_{j}}(\partial / \partial n)^{j} w(x), x \in \partial G$.

Application of this result for $j=1, j=2$, up to and including $j=k$, completes the proof.

Lemma IV.3a. Let there be given functions $B^{a_{1},-, a_{j}} \in C^{j}(B(0, b)), 0 \leq j \leq k$, that are symmetric in the indices. If the integral

$$
\int_{\sigma(0, b)} \sum_{j=0}^{k} p_{, a_{1}, \ldots, a_{j}} B(x)^{a_{1}, \ldots, a_{j}} d S,
$$

vanishes identically for all functions $p \in C_{0}^{k^{\prime}}(B(0, b))$, for some $k^{\prime} \geq k$, then

$$
\sum_{i=0}^{k-j}(-1)^{i}\left(\begin{array}{c}
i+j \\
i
\end{array}\right) B_{, a_{j+1}^{\prime}, \ldots, a_{j+i}^{\prime}}^{\nu, \nu a_{j+1}^{\prime},-, a_{j+i}^{\prime}}=0 \quad\left(j \nu^{\prime} s\right)
$$

for $x \in \sigma(0, b), 0 \leq j \leq k$.

Conversely, (3.2) implies that (3.1) vanishes for all $p \in C_{0}^{k^{\prime}}(B(0, b))$.

Proof. The functions $B^{a_{1},-, a_{j}}, 0 \leq j \leq k$, are symmetric. As $p \in C^{k^{\prime}}(B(0, b))$, also the derivatives $p, a_{1},-, a_{j}, 0 \leq j \leq k$, are symmetric in the indices. After a simple rearrangement, using this symmetry, the integrand of (3.1) can be written as

$$
\left.\sum_{j=0}^{k} \sum_{i=0}^{k-j}\left(\begin{array}{c}
i+j \\
i
\end{array}\right) p, \nu, \ldots, \nu a_{j+1}^{\prime}, \ldots, a_{j+i}^{\prime} B(x)^{\nu,-\nu a_{j+1}^{\prime}, \ldots, a_{j+i}^{\prime}} \quad \text { (j } \nu^{\prime} s\right) \text {. }
$$

After identifying $\mathbf{n}$ with $(0,-, 0,1)$ this expression can be written as

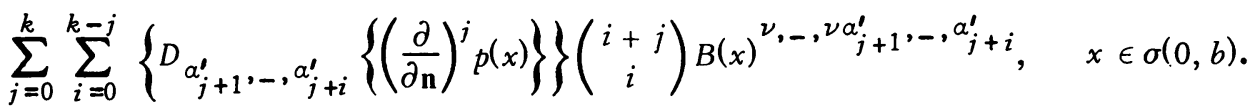

Using this expression, and the fact that the functions $\left(\partial / \partial_{n}\right)^{j} p, 0 \leq j \leq k$, have compact support in $\sigma(0, b)$, one obtains from an integration by parts that (3.1) is equal to 


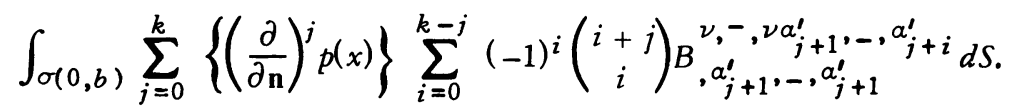

From Lemma IV.1, it follows that the functions $(\partial / \partial \mathrm{n})^{j} p, 0 \leq j \leq k$, can be chosen arbitrarily and independently in $C_{0}^{k^{\prime}-j}(\sigma(0, b))$. Using this, one obtains the first part of the lemma from a variational argument. The second part follows from substitution of (3.2) into (3.3).

Lemma IV.3b. Let there be given functions $B(x)^{\alpha_{1},-, \alpha_{j}} \in C^{j}$ (nbh $\left.\partial G\right), 0 \leq$ $j \leq k$, that are symmetric in the indices and assume that $\partial G \in C^{k^{\prime}}$, for some $k^{\prime} \geq k$. If the integral

$$
\int_{\partial G} \sum_{j=0}^{k} p_{, a_{1}, \ldots, a_{j}} B(x)^{a_{1}, \ldots, a_{j}} d S,
$$

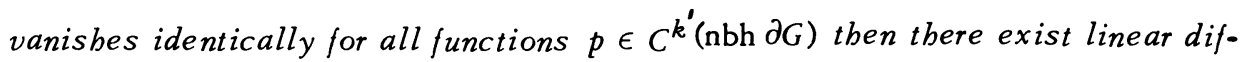
ferential expressions

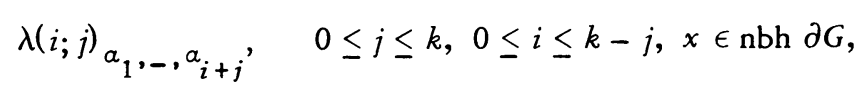

of order $i$, such that

$$
\sum_{i=0}^{k-j} \lambda(i ; j)_{a_{1}, \ldots, a_{i+j}} B(x)^{\alpha_{1},-, a_{i+j}}=0, \text { for } x \in \partial G, 0 \leq j \leq k .
$$

Conversely, (3.6) implies that (3.4) vanishes for all $p \in C^{k^{\prime}}$ (nbh $\partial G$ ).

Proof. Let $x_{b}$ be a point of $\partial G$. As $\partial G \in C^{k^{\prime}}$, there exists an open neighborhood of $x_{b}, N\left(x_{b}\right)$, and a one-to-one mapping $x=x(y)$, of $N\left(x_{b}\right)$ onto $B(0,1)$, that is together with its inverse $y=y(x), k^{\prime}$-times continuously differentiable, and such that $N\left(x_{b}\right) \cap \partial G$ is mapped onto $\sigma(0,1)$.

If $p \in C_{0}^{k^{\prime}}\left(N\left(x_{b}\right)\right)$, then $(3.4)$ reduces to

$$
\int_{N\left(x_{b}\right) \cap \partial G} \sum_{j=0}^{k} p_{, a_{1}, \ldots, a_{j}} B(x)^{\alpha_{1}, \ldots, a_{j}} d S .
$$

Now the coordinate transformation $x=x(y)$ is applied to (3.7). Then Lemma IV.3a is applied to the corresponding integral in $y$-coordinates. Finally the coordinate transformation $y=y(x)$ is applied to the identities corresponding to (3.2). After this transformation, the identities take the form

$$
\sum_{i=0}^{k-j} \lambda^{b}(i ; j)_{a_{1}, \ldots, a_{i+j}} B(x)^{\alpha_{1}, \ldots, a_{i+j}}=0, \quad x \in \partial G \cap N\left(x_{b}\right), 0 \leq j \leq k
$$

Here the expressions

$$
\lambda^{b}(i ; j)_{a_{1}, \ldots, a_{i+j}}, \quad 0 \leq j \leq k, 0 \leq i \leq k-j, x \in N\left(x_{b}\right),
$$


are linear differential expressions of order $i$. Thus one obtains from Lemma V.3a

(3.10) The integral (3.7) vanishes for all $p \in C_{0}^{k^{\prime}}\left(N\left(x_{b}\right)\right)$ iff (3.8) is satisfied.

As $\partial G$ is compact in $R^{\nu}$, there exists a finite number of points $\left\{x_{b}\right\}, 1 \leq b \leq$ $r, r<\infty$, with suitably chosen corresponding open neighborhoods $N\left(x_{b}\right)$, that together constitute a covering of $\partial G$, such that the procedure above can be carried out for all the $N\left(x_{b}\right)$ 's. Thus one obtains (3.8), for $1 \leq b \leq r$. The proof of the lemma is completed with the definition

$$
\lambda(i ; j)_{a_{1}, \ldots, a_{i+j}}=\lambda^{b}(i ; j)_{a_{1}, \ldots, a_{i+j}} \text { for } x \in M\left(x_{b}\right) .
$$

Here $\left\{M\left(x_{b}\right)\right\}, 1 \leq b \leq r$, is a disjoint covering of $\partial G$ that satisfies $\overline{M\left(x_{b}\right)} \subset N\left(x_{b}\right)$, for $1 \leq b \leq r$.

\section{The variational problem.}

(1) Definitions.

The differential expression $L$ is defined by

$$
L=\sum_{k=0}^{m} A(x)^{a_{1}, \cdots, a_{k}} D_{a_{1}} \cdots D_{a_{k}}, \quad x \in G .
$$

The coefficients are real and symmetric in the indices and $A(x)^{a_{1},-,{ }^{a} k} \in C^{k}(\bar{G})$, for $0 \leq k \leq m$.

The noncharacteristic part of the boundary of $G, \partial G^{\prime}$, is defined by (1.2).

$$
\partial G^{\prime}=\left\{x \in R^{\nu}: x \in \partial G \wedge n_{a_{1}} \cdots n_{a_{m}} A(x)^{a_{1},,_{m}} \neq 0\right\} .
$$

The functional $J$, defined on $C^{l}(\bar{G})$, is given by

$$
J(u)=\int_{G} F\left(x, u(x), D u(x),-, D^{l} u(x)\right) d V,
$$

with $l \in[1,-, m], F \in C^{2}\left(R^{\eta}\right), \eta=1+\Sigma_{k=0}^{l}\left(\nu^{k}\right)$ and $\partial F / \partial u, a_{1} ;-, a_{j}$ is symmetric in $\alpha_{1},-, \alpha_{j}$ for $2 \leq j \leq l$. As $u$ is chosen in $C^{l}(\bar{G})$, the last assumption is made without loss of generality.

For a given function $f \in C(G)$, the function spaces $U, U_{H}, U^{(i)}$ and $U_{H}^{(i)}$ are defined by

$$
\begin{aligned}
U & =\left\{u \in C^{m}(G): L u=f \wedge J(u) \text { is defined }\right\}, \\
U_{H} & =\left\{u \in C^{m}(G): L u=0\right\} . \\
U^{(i)} & =U \cap C^{i}(\bar{G}) ; \quad U_{H}^{(i)}=U_{H} \cap C^{i}(\bar{G}) .
\end{aligned}
$$

The class of admissible functions $W$ and the corresponding class of admissible variations $W_{H}$ are chosen in accordance with

$$
W_{C} U \text { is an affine subspace of } C^{m}(G) \text {, }
$$

$$
W_{H}=\left\{\delta u \in C^{m}(\bar{G}): \delta u=u_{1}-u_{2} \wedge u_{1}, u_{2} \in W\right\} \text {. }
$$


Remark. If $u \in W$ and $\delta u \in W_{H}$, then it follows from (1.6) that $L(\delta u(x))=0$, $x \in G$, and that $u+\delta u \in W$.

The variation of $J$ at $u \in W$, with respect to $\delta u \in W_{H}$, is defined by

$$
J J(u ; \delta u)=J(u+\delta u)-J(u) .
$$

Finally, the definition of a relative extremum is

(1.8) The functional $J$ has a relative extremum $w$ ithin the class of admissible functions $W$ if there is a positive real number $\delta$ such that $\Delta J(u ; \delta u)$ is either definite positive (relative minimum), or definite negative (relative maximum), for all $\delta u \in W_{H}$ that satisfy $\|\delta u\|_{l}$ exists and is smaller than $\delta$.

(2) The principal linear part of the variation.

Lemma V.1. Let $F \in C^{2}\left(R^{\eta}\right) ; u \in W ; W \in U^{(l)} ; \delta u \in W_{H}$, then the principal linear part of the variation of $J$ at $u$ with respect to $\delta u$ is given by

$$
\delta J(u ; \delta u)=\int_{G} \sum_{k=0}^{l} \delta u_{, a_{1}, \ldots, a_{k}} \frac{\partial F}{\partial u_{, a_{1},-, a_{k}}} d V .
$$

If moreover $J$ has a relative extremum within $W$ for some $u \in U$ then $\delta J(u ; \delta u)=0$, for all $\delta u \in W_{H}$.

Proof. Applying the mean-value theorem to the integrand of $\Delta J(u ; \delta u)$ one obtains

$$
\begin{aligned}
\Delta J(u ; \delta u)= & \delta J(u ; \delta u)+R(u ; \delta u), \\
& \text { with } R(u ; \delta u)=\int_{G} \sum_{k=0}^{l} \delta u_{, a_{1}, \ldots, a_{k}} \frac{\partial(\bar{F}-F)}{\partial u_{, a_{1}, \ldots, a_{k}}} d V .
\end{aligned}
$$

Here the overbar indicates that the corresponding derivatives are to be evaluated, for $\left(x, u+\theta \delta u,-, D^{l}(u+\theta \delta u)\right), \theta \in(0,1)$. From (2.1) and (2.2) one obtains $\Delta J(u ; a \delta u)=a\{\delta J(u ; \delta u)+(1 / a) R(u ; a \delta u)\}$, for any real number $a$. As the functions

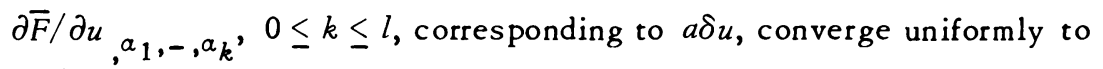
$\partial F / \partial u_{, a_{1},-, a_{\dot{k}}}$, as $a \rightarrow 0$, it follows that

$$
\lim _{a \rightarrow 0} \frac{1}{a} R(u ; a \delta u)=0 .
$$

Therefore $\delta J(u ; \delta u)$ is the linear principal part of $\Delta J(u ; \delta u)$. If $J$ has a relative extremum within $W$ for $u \in W$, then there exists an $\epsilon>0$, such that $\Delta J(u ; a \delta u)$ has the same sign for all $a \epsilon(-\epsilon,+\epsilon)$. It follows from (2.3) that this can only be true if $\delta J(u ; \delta u)=0$, for that $u$ and any $\delta u \in \delta W$. This completes the proof.

Lemma V.2. If $F \in C^{l+1}\left(R^{\eta}\right) ; u \in C^{2 l}(\bar{G}) ; \delta u \in C^{l}(\bar{G}) ;$ and $\partial G \in C^{1}$, then $\delta J(u ; \delta u)$ can be transformed into 


$$
\int_{G} \delta u(x)[F], u d V+\int_{\partial G} \sum_{j=0}^{l-1} \delta u_{, a_{1},-, a_{j}} Q^{a_{1,-, a_{j}}} d S .
$$

Here $[F],{ }_{,}$, the Euler-expression, is given by

$$
[F]_{, u}=\sum_{k=0}^{l}(-1)^{k} D_{a_{1}} \cdots D_{a_{k}}\left(\frac{\partial F}{\partial u_{, a_{1}, \ldots, a_{k}}}\right), \quad x \in G,
$$

and the expressions $Q^{a_{1},-, a_{j}}, 0 \leq j \leq l-1$, defined on $\partial G$, are given by

$$
Q^{a_{1},-, a_{j}}=\sum_{k=j+1}^{l}(-1)^{k-j-1}\left\{D_{a_{j+1}} \cdots D_{a_{k-1}}\left(\frac{\partial F}{\partial u_{, \alpha_{1}, \ldots, a_{k}}}\right)\right\} n_{a_{k}}
$$

Proof. Let $\Omega$ be an open set in $R^{\nu}$ that contains $\bar{G}$. It follows from $\mathrm{H}$. Whitney [8, Lemma 2$]$, that $u$ can be continued into $C^{2 l}(\Omega)$ and that $\delta u$ can be continued into $C^{l}(\Omega)$. Therefore $\delta u, a_{1,-, x_{k}}$ and $\partial F / \partial u, a_{1,-, a_{k}}$, both for $2 \leq k \leq l$, are symmetric in the indices. Thus one obtains as in (III.1.5), for $x \in \Omega$ and $k \in[1, l]$,

$$
\begin{aligned}
\delta u_{, a_{1}, \ldots, a_{k}} \frac{\partial F}{\partial u_{, a_{1}, \ldots, a_{k}}} & (-1)^{k} \delta u D_{a_{1}} \cdots D_{a_{k}}\left\{\frac{\partial F}{\partial u_{, a_{1}, \ldots, a_{k}}}\right\} \\
& +D_{a_{k}}\left\{\sum_{i=0}^{k-1}(-1)^{i}\left\{D_{a_{1}} \ldots D_{a_{i}}\left\{\frac{\partial F}{\partial u_{, a_{1}, \ldots, a_{k}}}\right\}\right\} \delta u_{, a_{i+1}, \ldots, a_{k-1}}\right\} .
\end{aligned}
$$

Hence, for $x \in \Omega$,

$$
\begin{aligned}
& \sum_{k=0}^{l} \delta u_{, a_{1}, \ldots, a_{k}} \frac{\partial F}{\partial u_{, a_{1}, \ldots, a_{k}}} \\
& \stackrel{(2.8)}{=} \delta u(x)[F]_{, u}+\sum_{k=1}^{l} D_{a_{k}}\left\{\sum_{i=0}^{k-1}(-1)^{i}\left\{D_{a_{1}} \ldots D_{a_{i}}\left\{\frac{\partial F}{\partial u_{, a_{1}, \ldots, a_{k}}}\right\}\right\} \delta u_{, a_{i+1}, \ldots, a_{k-1}}\right\} .
\end{aligned}
$$

After substitution of $(2.8)$ into $\delta J(u ; \delta u)$, as given in (2.1) and integrating by parts, one obtains

$$
\delta J(u ; \delta u)=\int_{G} \delta u(x)[F]_{, u} d V
$$

$$
+\int_{\partial G} \sum_{k=1}^{l} \sum_{i=0}^{k-1}(-1)^{i}\left\{D_{a_{1}} \cdots D_{a_{i}}\left\{\frac{\partial F}{\partial u_{, a_{1},-, a_{k}}}\right\}\right\} \delta u_{, a_{i+1}, \ldots, a_{k-1}} n_{a_{k}} d S .
$$

Afte: rearranging the summation this identity takes the form (2.4), with (2.6). This completes the proof. 
Lemma V.3. Assume that

(1 $\left.{ }^{\circ}\right) F \in C^{l+1}\left(R^{\eta}\right) ; u \in C^{2 l}(\bar{G}) ; \delta u \in U_{H}^{(m)} ; \partial G \in C^{m}$.

(2०) $A^{a_{1},-, a_{k}} \in C^{k}(\bar{G})$, for $0 \leq k \leq m$. $x \in G$.

$\left(3^{\circ}\right)$ There exists a function $\bar{v} \in \bar{C}^{m}(\bar{G})$ that satisfies $L^{*}(v(x))=[F], u$, for

Then, using the definitions (2.5) and (2.6), $\delta J(u ; \delta u)$ can be written as

$$
\begin{aligned}
\int_{\partial G}\left\{\sum_{j=0}^{l-1} \delta u_{1}, \ldots, a_{j}\left\{Q^{a_{1}, \ldots, a_{j}}-\sum_{i=0}^{m-j-1} v_{, a_{j+1}, \ldots, a_{j+i}} P(i ; j)^{a_{1}, \ldots, a_{i+j}}\right\}\right. \\
\left.-\sum_{j=l}^{m-1} \delta u_{, a_{1}, \ldots, a_{j}} \sum_{i=0}^{m-j-1} v_{, a_{j+1}, \ldots, a_{j+i}} P(i ; j)^{a_{1}, \ldots, a_{i+j}}\right\} d S .
\end{aligned}
$$

Proof. Under the above conditions, the following identity is valid.

$$
\int_{G} \delta u(x)[F], u d V=-\int_{G}\left\{v(x) L \delta u(x)-\delta u(x) L^{*} v(x)\right\} d V .
$$

By virtue of Green's identity, (III.1.3), the last expression is equal to

$$
-\int_{\partial G} \sum_{i=0}^{m-1} \sum_{j=0}^{m-i-1} v_{, a_{1}, \ldots, a_{i} \delta u_{, a_{i+1}, \ldots, a_{i+j}} P(i ; j)}{ }^{\alpha_{1}, \ldots, \alpha_{i+j}} d S .
$$

By virtue of the symmetry of $P(i ; j)^{\alpha_{1},-, a_{i+j}},(2.12)$ can be written as

$$
-\int_{\partial G} \sum_{j=0}^{m-1} \sum_{i=0}^{m-j-1} \delta u_{, \alpha_{1}, \ldots, a_{j}{ }^{v}, \alpha_{j+1}, \ldots, \alpha_{i+j}} P(i ; j){ }^{a_{1}, \ldots, a_{i+j}} d S .
$$

Substitution of this result into (2.4) completes the proof.

(3) The variational adjoint.

Definition. Assume that $F \in C^{l+1}\left(R^{\eta}\right) ; u \in U^{(2 l)} ; \partial G \in C^{m} ; A^{\alpha_{1},-, a_{k}} \in C^{k}(\bar{G})$, for $0 \leq k \leq m$. If there exists a function $v \in C^{m}(\bar{G})$ that satisfies

(3.1) $L^{*}(v(x))=[F], u, x \in G$, and

(3.2) $\delta J\left(u ; \delta u\right.$ ), as given in (2.10), vanishes for all functions $\delta u \in C^{m}$ (nbh $\partial G$ ), then this function $v$ is called a variational adjoint of $u$ with respect to $L$ and $J$.

Definition. The boundary condition (3.2), or conditions that are equivalent with (3.2) are called the variational adjoint boundary conditions.

Theorem V.1. If $v$ is a variational adjoint of $u$, with respect to $L$ and $J$, then the variational adjoint boundary conditions on $\partial G^{\prime}$ are given by (i), (ii) and (iii) as follows:

$$
(\partial / \partial \mathbf{n})^{j} v(x)=0
$$

for $0 \leq j \leq m-l-1$ and $x \in \partial G^{\prime}$. (If $l=m$, then this condition is vacuous.)

(ii) $\left(\frac{\partial}{\partial \mathbf{n}}\right)^{m-l} v(x)=(-1)^{m-l}\left(n_{\alpha_{1}} \cdots n_{a_{m}} A(x)^{a_{1},,_{m}}\right)^{-1} n_{a_{1}} \cdots n_{a_{l-1}} Q^{a_{1}, \ldots, a_{l-1}}$, for $x \in \partial G^{\prime}$. 
(iii)

$$
\sum_{b=0}^{l-1-j} \lambda(b ; j)_{a_{1},-, a_{j+b}} \sum_{i=m-l}^{m-j-b-1} v_{, a_{j+b+1}, \ldots, a_{j+b+i}} P\left(i_{j ; j+b}\right)^{a_{1}, \ldots, a_{i+j+b}}
$$

$=\sum_{b=0}^{l-1-j} \lambda(b ; j)_{a_{1},-, a_{j+b}} Q^{a_{1},-, a_{j+b}}$,

for $x \in \partial G^{\prime}$ and $l-2 \geq j \geq 0$. (If $l=1$, then this condition is vacuous.) For $0 \leq j \leq l-2,0 \leq b \leq l-1-j, x \in \partial G$, the $\lambda(b ; j)_{a_{1},-, a_{j+b}}$ 's denote linear differential expressions of order $b$.

Corollary. If $v$ is a variational adjoint of $u$, with respect to $L$ and $J$ while $l=1$, then the variational adjoint boundary conditions on $\partial G^{\prime}$ are given by

(ii) $\quad(\partial / \partial \mathbf{n})^{m-1} v(x)=(-1)^{m-1}\left(n_{a_{1}} \cdots n_{a_{m}} A(x)^{a_{1},-, a_{m}}\right)^{-1} n_{\alpha_{1}}\left(\partial F / \partial u_{, a}{ }_{1}\right)$, for $x \in \partial G^{\prime}$.

Definition. Each of the conditions in Theorem V.1 or in the corollary is called variational adjoint boundary condition $k$ if the highest order of differentiation of $v$ in that condition is $k$. So $k=j$ in (i), $k=m-1$ in (ii) and $k=m-j-1$ in (iii).

Definition. Depending on $L$ and $\partial G$, a function $w$ is of class $B_{j}$ if it is of class $C^{m}(\mathrm{nbh} \partial G)$ and if $(\partial / \partial \mathrm{n})^{i} w(x)=0$, for $0 \leq i \leq j-1$ if $x \in \partial G^{\prime}$, and for $0 \leq i \leq m-1$ if $x \in \partial G \sim \partial G^{\prime}$.

Proof. From (III.1.4) one obtains

$$
P(i ; j)^{a_{1},-, a_{i+j}}=(-1)^{m-j-1} n_{a_{m}} A^{a_{1},-, a_{m}}, \text { for } i+j=m-1 \text {. }
$$

Hence

$$
n_{a_{1}} \cdots n_{a_{i+j}} P^{a_{1},-\alpha_{i+j}} \neq 0, \quad \text { for } i+j=m-1 \text { and } x \in \partial G^{\prime} .
$$

From Lemma IV.1 it follows directly that

(3.3) If $\delta u$ is restricted to the class $B_{j}$ then $(\partial / \partial \mathrm{n})^{j} \delta u$ still can be chosen arbitrarily within the class $C_{0}^{m-j}\left(\partial G^{\prime}\right)$.

Now variational adjoint boundary condition 0 is obtained as follows. Let $\delta u$ be restricted to $B_{m-1}$, then in consequence of Lemma IV.2,

$$
\begin{aligned}
& \delta u_{, a_{1},-, a_{i}}=0, \text { for } x \in \partial G, 0 \leq i \leq m-2 . \\
& \delta u_{, a_{1},-, a_{m-1}}=n_{a_{1}} \cdots n_{a_{m-1}}(\partial / \partial \mathbf{n})^{m-1} \delta u(x), \text { for } x \in \partial G .
\end{aligned}
$$

Substitution of (3.4) into (2.10) yields 
$\delta J(u ; \delta u)$

$$
=\int_{\partial G^{\prime}}\left\{\left(\frac{\partial}{\partial \mathbf{n}}\right)^{m-1} \delta u(x)\right\} v(x) n_{a_{1}} \cdots n_{a_{m-1}} P(0, m-1)^{a_{1},-, a_{m-1}} d S
$$

for $\delta u \in B_{m-1}$.

From the definition of the variational adjoint boundary conditions and from (3.3) it follows that $\delta J(u ; \delta u)$, as given in (3.5), must vanish for arbitrary $(\partial / \partial \mathbf{n})^{m-1} \delta u \in C_{0}^{1}\left(\partial G^{\prime}\right)$.

From a variational argument and from (3.2) one obtains that $v(x)=0$, for $x \in \partial G^{\prime}$. If $m-l \geq 2$, the variational adjoint boundary condition 1 is obtained as follows. Let $\delta u$ be restricted to $B_{m-2}$ then, in consequence of Lemma IV.2,

$$
\begin{aligned}
& \delta u_{, a_{1}, \ldots, a_{i}}=0 \text { if } x \in \partial G \sim \partial G^{\prime}, 0 \leq i \leq m-1 \text {, } \\
& \text { if } x \in \partial G^{\prime}, 0 \leq i \leq m-3 \text {, } \\
& \delta u_{, \alpha_{1},-, a_{m-2}}=n_{a_{1}} \cdots n_{a_{m-2}}(\partial / \partial \mathbf{n})^{m-2} \delta u(x), \quad x \in \partial G^{\prime}, \\
& v(x)=0, \quad x \in \partial G^{\prime} \\
& v_{, a_{m-1}}=n_{a_{m-1}}(\partial / \partial \mathbf{n}) v(x), \quad x \in \partial G^{\prime}
\end{aligned}
$$

Substitution of (3.6) into (2.10) yields

$$
\begin{aligned}
& \delta J(u ; \delta u) \\
& =\int_{\partial G^{\prime}}\left\{\left(\frac{\partial}{\partial \mathbf{n}}\right)^{m-2} \delta u(x)\right\}\left\{\left(\frac{\partial}{\partial \mathbf{n}}\right) v(x)\right\} n_{a_{1} \cdots n_{a_{m-1}} P(1 ; m-2)^{a_{1},-, a_{m-1}} d S,}
\end{aligned}
$$

for $\delta u \in B_{m-2}$.

Now $\delta J(u ; \delta u)$ must vanish for arbitrary $(\partial / \partial \mathbf{n})^{m-2} \delta u(x) \in C_{0}^{2}\left(\partial G^{\prime}\right)$. From a variational argument and from (3.2) one obtains that $(\partial / \partial \mathrm{n}) v(x)=0$, for $x \in \partial G^{\prime}$. By repetition of this process one obtains also the variational adjoint boundary conditions $2,-, m-l-1$. Therefore

$$
(\partial / \partial \mathbf{n})^{j} v(x)=0, \quad \text { for } 0 \leq j \leq m-l-1, x \in \partial G^{\prime} .
$$

The variational adjoint boundary condition $m-l$ is obtained as follows. Let $\delta u$ be restricted to $B_{l-1}$ then one obtains from Lemma IV.2

$$
\begin{aligned}
& \delta u_{, a_{1}, \ldots, a_{i}=0} \quad \text { if } x \in \partial G \sim \partial G^{\prime}, 0 \leq i \leq m-1, \\
& \quad \text { if } x \in \partial G^{\prime}, 0 \leq i \leq l-2, \\
& \delta u_{, a_{1}, \ldots, a_{l-1}=} n_{a_{1}} \cdots n_{a_{l-1}}(\partial / \partial \mathbf{n})^{l-1} \delta u(x), \quad x \in \partial G^{\prime}, \\
& v_{, a_{1}, \ldots, a_{i}=0, \quad 0 \leq i \leq m-l-1, x \in \partial G^{\prime},} \\
& v_{, a_{l, \ldots, a_{m-1}}=} n_{a_{l}} \cdots n_{a_{m-1}}(\partial / \partial \mathbf{n})^{m-l} v(x), \quad x \in \partial G^{\prime} .
\end{aligned}
$$


Substitution of (3.9) into (2.10) yields

$\delta J(u ; \delta u)=\int_{\partial G^{\prime}}\left\{\left\{\left(\frac{\partial}{\partial \mathbf{n}}\right)^{l-1} \delta u(x)\right\} n_{a_{1}} \cdots n_{a_{l-1}}\right.$

$$
\text { - } \left.\left\{Q^{a_{1,-,} a_{l-1}}-\left\{\left(\frac{\partial}{\partial \mathbf{n}}\right)^{m-l} v(x)\right\} n_{a_{1}} \cdots n_{a_{m-1}} P(m-l ; l-1)^{a_{1},-, a_{m-1}}\right\}\right\} d S \text {. }
$$

Now $\delta J(u ; \delta u)$, as given in (3.10), must vanish for arbitrary choice of $(\partial / \partial n)^{l-1} \delta u$ $\epsilon C_{0}^{m-1+1}\left(\partial G^{\prime}\right)$. Therefore one obtains from a variational argument that (also set III(1.4))

(3.11) $(\partial / \partial \mathbf{n})^{m-l} v(x)=(-1)^{m-l}\left\{n_{\alpha_{1}} \cdots n_{a_{m}} A^{\alpha_{1},-, a_{m}}\right\}^{-1} n_{a_{1}} \cdots n_{a_{l-1}} Q^{a_{1},-, a_{l-1}}$, $x \in \partial G^{\prime}$.

If $l=1$, the proof is complete. If $l \geq 2$, (iii) remains to be proven. This is done as follows. From (3.8) and Lemma IV.2, one obtains $v_{, a_{1},-, a_{i}}=0$, for $0 \leq i \leq m-l-1$ and $x \in \partial G^{\prime}$. After letting $\delta u$ satisfy $(\partial / \partial \mathbf{n})^{j} \delta u \in C^{m-j}(\partial G)$, $0 \leq j \leq m-1$, and $(\partial / \partial \mathbf{n})^{j} \delta u(x)=0$, for $x \in \partial G \sim \partial G^{\prime}, 0 \leq j \leq m$; one obtains from the same lemma $\delta u, a_{1},-, a_{i}=0,0 \leq i \leq m-1$ and $x \in \partial G \sim \partial G^{\prime}$. From substitution of these results into (2.10) and from the assumption that $v$ is a variational adjoint of $u$ it follows that

$$
\int_{\partial G^{\prime}} \sum_{j=0}^{l-1} \delta u_{, a_{1}, \ldots, a_{j}}\left\{Q^{a_{1}, \ldots, a_{j}}-\sum_{i=m-l}^{m-j-1} v_{, a_{j+1}, \ldots, a_{j+i}} P(i ; j)^{a_{1}, \ldots, a_{i+j}}\right\} d S
$$

vanishes for all $\delta u \in C^{m}($ nbh $\partial G)$ that satisfy $(\partial / \partial n)^{j} \delta u(x)=0$, for $0 \leq j \leq m-1$ and $x \in \partial G \sim \partial G^{\prime}$.

If $\partial G=\partial G^{\prime}$, then (iii) follows directly from application of Lemma IV.3b to (3.12). A lemma comparable to Lemma IV.3b can be derived for the case where the domain of integration is a relatively open subset $S$ of $\partial G$ and where the function $p$ satisfies besides $p \in C^{k^{\prime}}$ (nbh $\partial G$ ) also the conditions $(\partial / \partial \mathbf{n})^{j} p(x)=g_{j}(x)$, $0 \leq j \leq k, x \in \partial G \sim S$, with given functions $g_{j}(x)$ of class $C^{k^{\prime}-j}(\partial G \sim S)$. The proof of this lemma is analogous to that of Lemma IV.3b. This lemma is used in order to establish (iii) for the case where $\partial G \neq \partial G^{\prime}$. This completes the proof.

VI. Necessary conditions for the optimization problem associated with a uniformly strongly elliptic partial differential equation.

(1) Definitions and existence theorem.

\section{Definition.}

(1.1) The differential expression $L$, as defined in (V.1.1) is called uniformly strongly elliptic in $\bar{G}$ if the coefficients are bounded in $\bar{G}$ and if 


$$
(-1)^{m / 2} A(x)^{a_{1},-a_{m}} \xi_{a_{1}} \cdots \xi_{a_{m}} \geq c\left\{\sum_{i=1}^{\nu}\left(\xi_{i}\right)^{2}\right\}^{m / 2},
$$

for $x \in \bar{G}$, some positive constant $c$ and arbitrary real $\nu$-vector $\xi$. (This definition implies that $m$ is even.)

The following existence theorem, associated with the classical Dirichlet problem for a strongly elliptic partial differential equation, follows directly from [3, Theorem 17.3]; [3, Theorem 13.1]; [3, Theorem 14.6]; [3, Lemma 13.3] (all in part I), and Lemma IV.1.

Theorem VI.1. Let the following conditions be satisfied for some integer $p$, $p \geq 0$, and $t=p+[\nu / 2]+1$.

$\left(1^{\circ}\right) G$ is bounded and $\partial G \in C^{m+t}$.

$\left(2^{\circ}\right)$ L is uniformly strongly elliptic in $\bar{G}$.

(3०) $A^{a_{1},-, a_{j}} \in C^{j+t}(\bar{G}), 0 \leq j \leq m$.

$\left(4^{\circ}\right) f \in C^{t}(\bar{G})$.

(5) $g_{j} \in C^{m+t-j}(\partial G)$, for $0 \leq j \leq m / 2-1$.

Then the Fredholm alternative bolds for the classical Dirichlet problem

$$
\begin{aligned}
& L(u(x))=f(x), \quad x \in G, \\
& (\partial / \partial \mathbf{n})^{j} u(x)=g_{j}(x), \quad x \in \partial G, 0 \leq j \leq m / 2-1,
\end{aligned}
$$

while any solution of (1.2) is of class $C^{m+p}(\bar{G})$.

(2) Necessary conditions.

Theorem VI.2. Assume that (1.2) is uniquely solvable and

$\left.\left.\left(1^{\circ}\right) l \in[1,1 / 2 m], q \geq \max \right\} 0,2 l+[\nu / 2]+1-m\right\}$.

$\left(2^{\circ}\right)$ The requirements $\left(1^{\circ}\right),\left(2^{\circ}\right)$, and $\left(4^{\circ}\right)$ of Theorem VI.1 are met with $p=q$, and requirement $\left(3^{\circ}\right)$ is replaced with $A^{a_{1},-, a_{j}} \in C^{2 j+t}(\bar{G}), 0 \leq j \leq m$.

( $\left.3^{\circ}\right) F \in C\left(R^{\eta}\right)^{l+[\nu / 2]+2}$.

$\left(4^{\circ}\right) W=U^{(m+q)}$.

If now $u \in W$, then the boundary value problem (2.1) admits one solution which is of class $C^{m}(\bar{G})$.

$$
\begin{aligned}
& L^{*}(v(x))=[F], u, \quad x \in G, \\
& (\partial / \partial \mathbf{n})^{j} v(x)=0, \quad 0 \leq j \leq 1 / 2 m-1, x \in \partial G .
\end{aligned}
$$

If moreover $\delta J(u ; \delta u)=0$, for all $\delta u \in W_{H}$, then the solution of (2.1) also satisfies the variational adjoint boundary conditions $1 / 2 m,-, m-1 .\left(\partial G=\partial G^{\prime}.\right)$ Conversely, if there exists a solution of (2.1) that satisfies the variational adjoint boundary conditions $1 / 2 m,-, m-1$, then $\delta J(u ; \delta u)=0$, for all $\left.\delta u \in W_{H^{*}}{ }^{2}\right)$

(2) The corresponding theorem for nonuniquely solvable Dirichlet problem in [9] contains an error ('then any' should be 'then (4.1) is solvable and any'). The correct formulation and proof are given in [10]. 
Definition. If the requirements $\left(1^{\circ}\right),-,\left(4^{\circ}\right)$ of Theorem VI. 2 are met, then the associated boundary value problem is defined by

$$
\begin{aligned}
& L(u(x))=f(x), \quad u \in W, x \in G, \\
& L^{*}(v(x))=[F], u, \quad x \in G,
\end{aligned}
$$

the variational adjoint boundary conditions $0,-, m-1$,

as given in Theorem V.1 are satisfied on $\partial G$.

Corollary. If $J$ bas a relative extremum within $W$, for some $u \in W$ and if the requirements of Theorem VI.2 are met, then there exists precisely one function $v \in C^{m}(\bar{G})$ such that $u$ and $v$ together satisfy the associated boundary value problem.

Proof. It can easily be verified that $\left(1^{\circ}\right),\left(2^{\circ}\right)$ and $\left(3^{\circ}\right)$ are chosen such that Theorem VI.1 establishes the existence and smoothness of solutions of (2.1).

Under the conditions $\left(1^{\circ}\right),-,\left(4^{\circ}\right),(2,1)$, one obtains from Lemma V.3 that $\delta J(u ; \delta u), \delta u \in W_{H}$, can be written as in (V.2.10). The strong ellipticity condition implies that $\partial G=\partial G^{\prime}$. It follows from Theorem VI.1 that the solutions of

$$
\begin{aligned}
& L(\delta u(x))=0, \text { for } x \in G, \\
& (\partial / \partial \mathbf{n})^{j} \delta u(x)=g_{j}(x), \text { for } 0 \leq j \leq 1 / 2 m-1, \text { and } x \in \partial G,
\end{aligned}
$$

where the functions $g_{j}, 0 \leq j \leq 1 / 2 m-1$, can be chosen arbitrarily in $C(\partial G)^{m+t-j}$, are elements of $W_{H}$.

It is observed that in (2.1) the variational adjoint boundary conditions $0,-, 1 / 2 \mathrm{~m}$ are chosen. The rest of the proof of Theorem VI. 2 is analogous to the last part of the proof of Theorem V.1, starting with the derivation of the variational adjoint boundary condition $1 / 2 m$ from the arbitrariness of $(\partial / \partial n)^{m / 2-1} \delta u(x)$ on $\partial G$. The proof of the corollary follows directly from Lemma V.1 and the theorem. This completes the proof.

VII. Necessary conditions for the optimization problem associated with a uniformly parabolic partial differential equation.

(1) Definitions and existence theorem. With regard to the domain $G$ the following definitions are in force throughout this chapter. (It is recalled that $x^{\prime}=$ $\left(x_{1},-, x_{\nu-1}\right)$.)

(1.1) $G_{\nu_{-}}$is an open domain in $R^{\nu-1}$ with boundary $\partial G_{\nu_{-}} \cdot H_{\nu_{-}}$is the intersection of the $R^{\nu-1}$-closure of $G_{\nu-1}$ with an open $R^{\nu-1}$-neighborhood of $\partial G_{\nu_{-1}} . G=G_{\nu_{-1}} \times(0, T) . \partial G$ is the boundary of $G . \partial G(0)=\left\{x \in R^{\nu}: x^{\prime} \in G_{\nu_{-1}} \wedge\right.$ $\left.x_{\nu}=0\right\}$. Similar for $\partial G(T), \partial H(0)=\left\{x \in R^{\nu}: x^{\prime} \in H_{t_{-1}} \wedge x_{\nu}=0\right\}$. Similar for $\partial H(T), \partial G(0, T]=\left\{x \in R^{\nu}: x^{\prime} \in \partial G_{\nu-1} \wedge x_{\nu} \in(0, T]\right\}$.

The differential expression $L$ in this chpater is defined by 


$$
L=D_{\nu}+L^{\prime}, \text { with } L^{\prime}=\sum_{k=0}^{m} A(x)^{\alpha_{1}^{\prime}, \cdots, a_{k}^{\prime}} D_{\alpha_{1}^{\prime}} \ldots D_{\alpha_{1}^{\prime}}, \quad x \in \bar{G} .
$$

Definition. (1.3) $L$ is called uniformly parabolic in $\bar{G}$ if the coefficients are bounded in $\bar{G}$ and if

$$
(-1)^{m / 2} A(x)^{a_{1}^{\prime},-, a_{m}^{\prime}} \xi_{a_{1}^{\prime}} \cdots \xi_{a_{m}^{\prime}} \geq c\left\{\sum_{i=1}^{\nu-1}\left(\xi_{i}\right)^{2}\right\}^{m / 2},
$$

for $x \in \bar{G}$, some positive constant $c$ and arbitrary real $(\nu-1)$-vector $\xi$. (This definition implies that $m$ is even.)

In order to be able to use previously derived formulas, from now on $L$ will be written as

$$
L=\sum_{k=0}^{m} A(x)^{a_{1}, \ldots,{ }^{a} D_{a_{1}}} \cdots D_{a_{k}}, \quad x \in \bar{G},
$$

with $A(x)^{\alpha_{1},-, \alpha_{k}} \equiv 0$, if at least one index has value $\nu$ and $k \in[2, m]$, while $A^{\nu}=1$.

The following existence theorem follows directly from [3, part 2, Theorem 9.1 and the second corollary on p. 141].

Theorem VII.1. Let the following conditions be satisfied for some nonnegative integer $p$.

$\left(1^{\circ}\right) r=1+[(2 p+\nu+1) / 2 m] ; s=m+p+r+[\nu / 2] ; t=s-r+m(r+1)$.

(2०) $G_{\nu-1}$ and $T$ are bounded; $\partial G_{\nu-1} \in C^{m(r+1)}$.

(3) $L$ is uniformly parabolic in $\bar{G}$.

(4º) $A^{a_{1},-, \alpha_{j}} \in C^{s}(\bar{G}), 0 \leq j \leq m$.

$\left(5^{\circ}\right) f \in C^{t}(\bar{G}) ;(\partial / \partial n)^{j} f(x)=0$, for $0 \leq j \leq t$ and $x \in \partial H(0)$.

$\left(6^{\circ}\right) w \in C^{t+m}(\bar{G}) ; w(x) \equiv 0$, if $x \in \partial H(0) \times[0, \epsilon)$, for some $\epsilon>0$.

Then the intial boundary value problem

$$
\begin{aligned}
& L u(x)=f(x), \quad x \in G \cup \partial G(T), \\
& (\partial / \partial \mathbf{n})^{j} u(x)=(\partial / \partial \mathbf{n})^{j} w(x), \quad 0 \leq j \leq m / 2-1, x \in \partial G(0, T], \\
& u(x)=w(x), \quad x \in \partial G(0),
\end{aligned}
$$

admits a unique solution which is of class $C^{m+p}(\bar{G})$.

(2) Necessary conditions.

Theorem VII.2. Assume that

(1 $\left.{ }^{\circ}\right) l \in[1,1 / 2 m], q \geq t(0)+2 l-m$, where $t(0)=3 m+[\nu / 2]+m[(\nu+1) / 2 m]$.

$\left(2^{\circ}\right)$ The requirements $\left(1^{\circ}\right),\left(2^{\circ}\right),\left(3^{\circ}\right)$ and $\left(5^{\circ}\right)$ of Theorem VII. 1 are met with $p=q$, while requirement $\left(4^{\circ}\right)$ is replaced by $A(x)^{\alpha_{1},-, \alpha_{j}} \in C(\bar{G})^{j+s}, 0 \leq j \leq m$. 
(3०) $F \in C^{l+1+t(0)}\left(R^{\eta}\right)$.

(4) $W=U^{(m+q)}$.

$\left(5^{\circ}\right)(\partial / \partial \mathbf{n})^{j}[F], u=0$, for $0 \leq j \leq t(0), x \in \partial H(0)$, and any $u \in W$.

$\left(6^{\circ}\right) \partial F / \partial u, \nu$ has compact support in $\partial G(T)$ for any $u \in W$.

( $\left.7^{\circ}\right) \partial F / \partial u_{, a_{1},-, a_{j}} \equiv 0$, for $2 \leq j \leq l$ and $x \in \operatorname{nbh} \partial G(0) \cup \operatorname{nbh} \partial G(T)$.

If now $u \in W$, then the initial boundary value problem (2.1) admits a unique solution, which is of class $C^{m}(\bar{G})$.

$$
\begin{aligned}
& L^{*} v(x)=[F], u, \text { for } x \in G ; \\
& (\partial / \partial \mathbf{n})^{j} v(x)=0, \text { for } 0 \leq j \leq m / 2-1 \text { and } x \in \partial G[0, T), \\
& v(x)=+\partial F / \partial u, \nu, \text { for } x \in \partial G(T) .
\end{aligned}
$$

If moreover $\delta J(u ; \delta u)=0$, for all $\delta u \in W_{H}$, then a solution of (2.1) also satisfies the variational adjoint boundary conditions $1 / 2 m,-, m-1$ on the lateral boundary, $\partial G(0, T)$ and the condition: $v(x)=+\partial F / \partial u, \nu$ on $\partial G(0)$. Conversely, if there exists a solution of (2.1) that also satisfies the se extra boundary conditions then $\delta J(u ; \delta u)$ vanishes for all $\delta u \in W_{H^{*}}$

Definition. If the requirements $\left(1^{\circ}\right),-,\left(7^{\circ}\right)$ of Theorem VII. 2 are met, then the associated boundary value problem is defined by

$$
\begin{aligned}
& L u(x)=f(x), \quad u \in W, x \in G, \\
& L^{*} v(x)=[F]_{, u}, \quad x \in G,
\end{aligned}
$$

the variational adjoint boundary conditions $0,-, m-1$,

as given in Theorem V.1, are satisfied on $\partial G(0, T)$,

$$
v(x)=+\partial F / \partial u, \nu, \text { for } x \in \partial G(0) \cup \partial G(T) .
$$

Corollary. If $J$ has a relative extremum within $W$, for some $u \in W$ and if the requirements of Theorem VII.2 are met, then there exists a function $v \in C^{m}(\bar{G})$, such that $u$ and $v$ together satisfy the associated boundary value problem (2.2).

Proof. It can easily be verified that the conditions $\left(1^{\circ}\right),-,\left(6^{\circ}\right)$ are chosen such that Theorem VII.1 establishes the existence and smoothness of the solution of (2.1).

From substitution of $\left(7^{\circ}\right)$, of $\mathbf{n}=(0,-, 0,-1)$ on $\partial G(0)$ and of $\mathbf{n}=(0,-, 0,+1)$ on $\partial G(T)$ into (V. 2.6) it follows that

$$
\begin{aligned}
Q^{a_{1,-,}{ }_{j}} & =0, \quad 1 \leq j \leq l-1, x \in \partial G(0) \cup \partial G(T) \\
Q & =-\partial F / \partial u, \nu \text { on } \partial G(0) ; \quad Q=\partial F / \partial u_{, \nu} \text { on } \partial G(T) .
\end{aligned}
$$

From substitution of the functions $A^{\alpha_{1},-, \alpha_{j}}$, as given in $(1.4)$, of $\mathbf{n}=(0,-,-1)$ on $\partial G(0)$ and of $\mathbf{n}=(0,-, 0,1)$ on $\partial G(T)$ into (III.1.4) it follows that 


$$
\begin{array}{r}
P(i ; j)^{a_{1},-, a_{i+j}}=0, \quad 1 \leq i+j \leq m-1, x \in \partial G(0) \cup \partial G(T) . \\
P(0,0)=-1, \quad \text { on } \partial G(0) ; \quad P(0,0)=1 \quad \text { on } \partial G(T) .
\end{array}
$$

Under the above conditions one obtains from Lemma V.3 and from substitution into (V.2.10), of the boundary conditions in (2.1), of (2.3) and of (2.4):

$$
\delta J(u ; \delta u)=\int_{\partial G(0)} \delta u(x)\left\{v(x)-\frac{\partial F}{\partial u}, \nu\right\} d S
$$

$$
+\int_{\partial G(0, T)}\left\{\sum_{j=0}^{l-1} \delta u_{, \alpha_{1}, \ldots, a_{j}}\left\{Q^{a_{1,-,} a_{j}}-\sum_{i=m / 2}^{m-j-1} v_{,_{j+1}, \ldots, a_{j+i}} P(i ; j)^{a_{1}, \ldots, a_{i+j}}\right\}\right.
$$

$$
\left.-\sum_{j=l}^{m-1} \delta u_{, \alpha_{1},-, \alpha_{j}} \sum_{i=m / 2}^{m-j-1} v_{, \alpha_{j+1},-, \alpha_{j+i}} P(i ; j)^{\alpha_{1,-,} \alpha_{i+j}}\right\} d S \text {. }
$$

First restrict $u$ to $W^{\prime}$,

(2.6) $W^{\prime}=\left\{u \in W:(\partial / \partial \mathbf{n})^{j}(u(x)-w(x))=0,0 \leq j \leq m / 2-1, x \in \partial G(0, T)\right\}$,

here $w$ is a fixed function in $W \cap C(\bar{G})^{t+m}$.

Now, $\delta u$, restricted to $\partial G(0)$, still can be chosen arbitrarily in $C_{0}(\partial G(0))^{t+m}$ while $\delta u_{, a_{1},-, a_{j}} \equiv 0,0 \leq j \leq m / 2-1, x \in \partial G(0, T)$. The last condition implies that the integral over $\partial G(0, T)$, in (2.5) vanishes for all $\delta u \in W_{H^{\prime}}^{\prime}$ Using a variational argument one obtains from $\delta J(u ; \delta u)$, as given in (2.5), vanishes for all $\delta u \in W_{H}^{\prime}$ :

$$
v(x)=\partial F / \partial u, \nu, \text { for } x \in \partial G(0) .
$$

Conversely, if (2.7) is satisfied then the integral over $\partial G(0)$ vanishes for all $\delta u \in W_{H}$.

Next, restrict $u$ to $W^{\prime \prime}$,

$$
W^{\prime \prime}=\{u \in W: u(x)=w(x), x \in \partial G(0)\}
$$

here $w$ is a given function in $W \cap C(\bar{G})^{t+m}$.

Now $(\partial / \partial \mathbf{n})^{j} \delta u(x), 0 \leq j \leq m / 2-1, x \in \partial G(0, T)$, still can be chosen arbitrarily in $C_{0}(\partial G(0, T))^{t+m-j}$, while $\delta u(x) \equiv 0$, for $x \cdot \epsilon \partial(0)$. The last condition implies that in (2.5) the integral over $\partial G(0)$ vanishes for all $\delta u \in W_{H}^{\prime \prime}$. At this point it is observed that, because of the Dirichlet conditions on $\partial G(0, T), \delta J(u ; \delta u)$, for $u \in W^{\prime \prime}$, has the same form as in the proof of Theorem VI.2, that the same variation possibilities exist and that $\partial G(0, T)$ is noncharacteristic. Therefore, from $\delta J(u ; \delta u)$, as given in $(2.5)$, vanishes for all $\delta u \in W_{H}^{\prime \prime}$ one obtains similar to in the proof of Theorem VI.2:

(2.9) The variational adjoint boundary conditions $1 / 2 m,-, m-1$, as given in Theorem V.1 are satisfied on $\partial G(0, T)$. 
And conversely, if (2.9) is satisfied, then the integral over $\partial G(0, T)$, in (2.5), vanishes for all $\delta u \in W_{H}$. This completes the proof of Theorem VII.2.

The corollary follows directly from Lemma V.1 and Theorem VII.2. With the same assumptions and definitions, it is clear from the proof of Theorem VII.2 that the next remark can be made.

Remark. If $W^{\prime}$ is taken for the class of admissible functions then the associated boundary value problem takes the form

(2.10) (2.2), with the variational adjoint boundary conditions $1 / 2 m,-, m-1$, $x \in \partial G(0, T)$ replaced by $(\partial / \partial \mathbf{n})^{j} u(x)=(\partial / \partial \mathbf{n})^{j} w(x), 0 \leq j \leq m / 2-1, x \in \partial G(0, T]$.

If $W^{\prime \prime}$.is taken for the class of admissible functions then the associated boundary value problem takes the form

(2.11) (2.2), with the condition $v(x)=\partial F / \partial u_{\nu}, x \in \partial G(0)$, replaced by $u(x)=$ $w(x), x \in \partial G(0)$.

(3) The associated boundary value problem for the beat equation. In this section the associated boundary value problem will be formulated for the case where $L$ is given by

$$
L=D_{\nu}-\Delta_{\nu-1}, \quad \text { with } \Delta_{\nu-1}=\sum_{i=1}^{\nu-1} D_{i}^{2}
$$

So $m=2 ; l=1$ and $n_{a_{1}} n_{a_{2}} A^{a_{1} a_{2}}=-1$, for $x \in \partial G(0, T)$. Using the corollary with Theorem V.1, the variational adjoint boundary value problem (2.1) takes the form

$$
\begin{aligned}
& u_{, \nu}-\Delta_{\nu-1} u(x)=f(x), \quad x \in G, \\
& v_{, \nu}+\Delta_{\nu-1} v(x)=-[F]_{, u}, \quad x \in G, \\
& v(x)=0, \quad x \in \partial G(0, T), \\
& v(x)=\partial F / \partial u, \nu, \quad x \in \partial G(0) \cup \partial G(T), \\
& (\partial / \partial \mathbf{n}) v(x)=n_{a_{1}} \partial F / \partial u_{, a_{j}}, \quad x \in \partial G(0, T) .
\end{aligned}
$$

If the conditions $\left(1^{\circ}\right),-,\left(6^{\circ}\right)$ of Theorem VII.2 are satisfied, if $u \in W$, and if $J(u)$ has an extremum within $W$ for this $u$ then (3.2) admits a unique solution for $v$. Furthermore, $v \in C^{2}(\bar{G})$.

\section{BIBLIOGRAPHY}

1. R. Delver, The optimization of a functional within the class of solutions of a partial differential equation, J. Engrg. Math. 3 (1969), 253-264. MR 41 \#2497.

2. - Variational problems within the class of solutions of a partial differential equation, $\mathrm{Ph}$. D. Thesis, University of California, Berkeley, Calif., 1971.

3. A. Friedman, Partial differential equations, Holt, Rinehart and Winston, New York, 1969.

4. - Optimal control for parabolic equations, J. Math. Anal. Appl. 18 (1967), 479-491. 
5. K. A. Lur'e, The Mayer-Bolza problem for multiple integrals: Some optimum problems for elliptic differential equations arising in magnetohydrodynamics, Topics in Optimization, Academic Press, New York, 1967, pp. 147-193. MR 35 \#1380.

6. J. L. Lions, Controle optimal de systèmes gouvernés par des équations aux dérivées partielles, Dunod; Gauthier-Villars, Paris, 1968; English transl., Die Grundlehren der math. Wissenschaften, Band 170, Springer-Verlag, Berlin and New York, 1971. MR 39 \#5920; MR 42 \#6395.

7. C. Miranda, Equazioni alle derivate parziali di tipo ellittico, Ergebnisse der Mathematik und ihrer Grenzgebiete, Heft 2, Springer-Verlag, Berlin, 1955; English transl., Springer-Verlag, Berlin and New York, 1970. MR 19, 421.

8. H. Whitney, Analytic extensions of differentiable functions defined in closed sets, Trans. Amer. Math. Soc. 36 (1934), 63-89.

9. R. Delver, Variational problems within the class of solutions of a partial differential equation, Bull. Amer. Math. Soc. 77 (1971), 1100-1105.

10. - Boundary and interior control for partial differential equations (to appear).

DEPARTMENT OF MATHEMATICS, UNIVERSITY OF CALIFORNIA, BERKELEY, CALIFORNIA 94720

Current address: Department of Mathematics, University of Toronto, Toronto, Canada 